\title{
The Inspiring Science Education project and the resources for HEP analysis by university students
}

\author{
Dimitris Fassouliotis ${ }^{1}$, Christine Kourkoumelis ${ }^{1}$, and Stylianos Vourakis ${ }^{1}$ \\ ${ }_{1}$ Physics Faculty and Institute of Accelerating Systems and Applications, University of Athens, Panepistimioupoli, \\ Ilissia 15771, Greece
}

\begin{abstract}
The Inspiring Science Education outreach project has been running for more than two years, creating a large number of inquiry based educational resources for high-school teachers and students. Its goal is the promotion of science education in schools though new methods built on the inquiry based education techniques, involving large consortia of European partners and implementation of large-scale pilots in schools. Recent hands-on activities, developing and testing the above mentioned innovative applications are reviewed. In general, there is a lack for educational scenaria and laboratory courses earmarked for more advanced, namely university, students. At the University of Athens for the last four years, the HYPATIA on-line event analysis tool has been used as a lab course for fourth year undergraduate physics students, majoring in HEP. Up to now, the course was limited to visual inspection of a few tens of ATLAS events. Recently the course was enriched with additional analysis exercises, which involve large samples of events. The students through a user friendly interface can analyse the samples and optimize the cut selection in order to search for new physics. The implementation of this analysis is described.
\end{abstract}

\section{Introduction}

The European Union (EU) has long recognized the decreasing interest of young people in science subjects and has been very supportive towards and promoting outreach projects which would bridge the gap between school science education and active research. An effective way to stimulate students' interest towards STEM subjects is to actively involve them in hands-on top level research.

The authors of this contribution have been involved in several EU outreach projects for more than a decade. Some are already finished: Learning with ATLAS@CERN (2009-2011) [1], PATHWAY to inquiry based science education [2] and Discover the COSMOS (2010-2012) [3]. Three more are ongoing, Go-lab [4], Inspiring Science Education [5], which will be discussed in the next session and CREATIONS which started in October 2015.

The finished projects have already created a large number of resources addressed mainly to teachers, which are classified according to subject (keywords), age, difficulty, duration and language. The Discover the COSMOS portal [6] contains about 95,000 science education learning objects and activities connected to the science curriculum from Astronomy, Space Physics and HEP.

All projects followed the Inquiry Based pedagogical framework. Instead of just delivering lectures to the students, they were encouraged to learn by making hypothesis, perform interactive investigation to verify the hypothesis and draw conclusions by themselves, which were then discussed in the class. 
The authors have been involving both high school as well as university students in activities which simulate the work of the researches at CERN. These are the subjects of the present contribution.

\section{The Inspiring Science Education project and the HYPATIA demonstrator}

The Inspiring Science Education (ISE) project began in April 2013 and will last until July 2016. The ISE partners design, plan and implement large-scale pilots to stimulate and evaluate innovative use of existing eLearning tools and digital resources for scientific disciplines and technology (STEM related subjects), enhancing science learning in 5,000 primary and secondary schools in 14 European Countries. During its first two years of operation the project has already reached 2,700 schools in Europe. In line with the ISE's inquiry learning framework, the partners have been designing the so called ISE demonstrators which are a series of success stories making effective use of a wide range of eLearning tools and applications. Each demonstrator is a complete lesson plan structured in several phases: Orienting and Asking Questions, Hypothesis Generation and Design, Planning and Investigation, Analysis and Interpretation and Conclusion and Evaluation. In order to evaluate their benefits and effectiveness, each phase of the demonstrator is accompanied by assessment questions (adopted according to the PISA 2012 Problem Solving Competence Framework) [7]. Currently there are 120 demonstrators. In addition to the demonstrators, ISE has harvested existing repositories with 278,000 educational resources (mainly from Open Discovery Space [8] and from Discover the COSMOS portal).

The demonstrator which is designed to provoke students' curiosity for HEP (which in most countries is absent from the national school curricula) is the HYPATIA demonstrator [9]. This has been built according to the various phases of Inquiry learning referred above and shown on Fig.1.

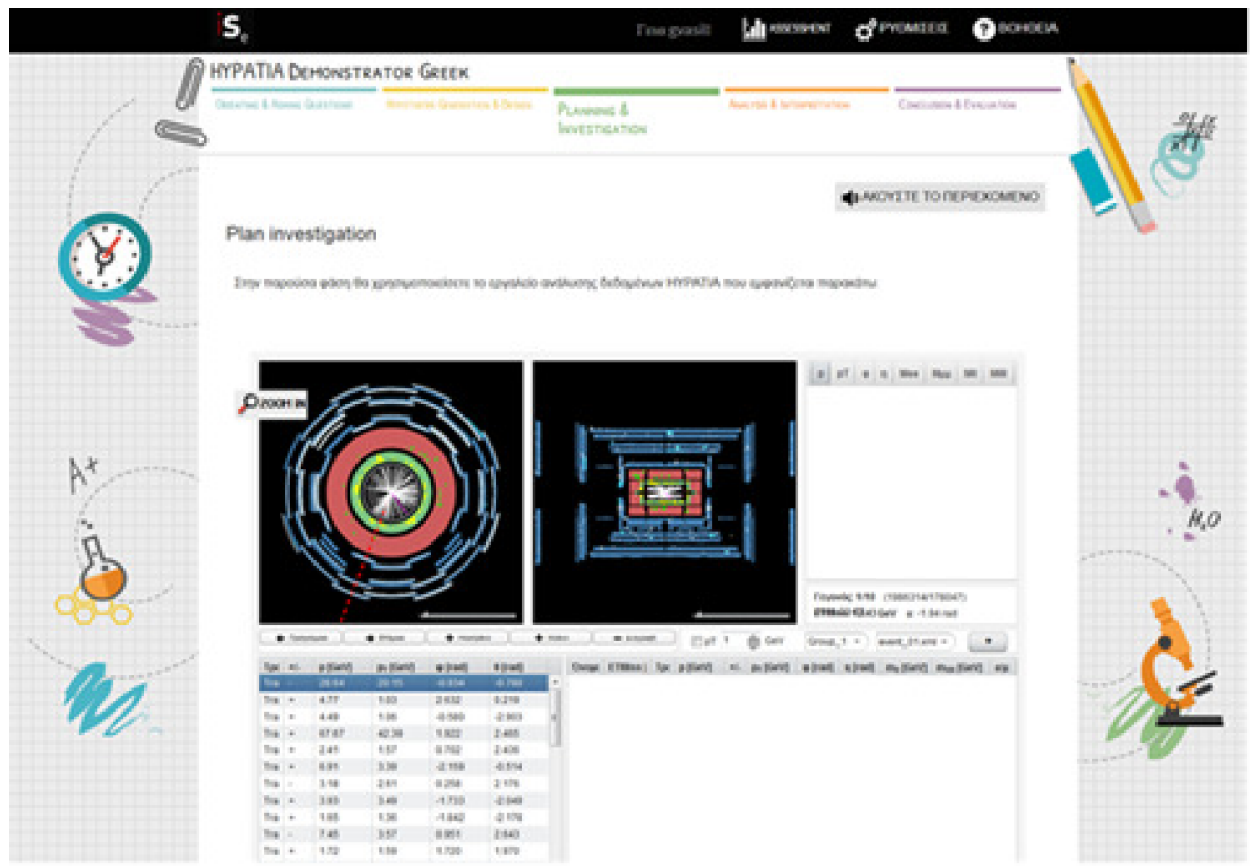

Figure 1. Screenshot of the HYPATIA demonstrator. 
Special effort has been made during the design process in order to achieve the following goals:

- Engage the students in hands-on experimentation directly connected to the top-level research at CERN by analysing data almost in real-time

- The material can fit in the duration of a school lesson and is directly connected to the Big ideas of science

- The material has also been adapted to the age of students

Moreover, as previously mentioned, during the step-by-step students' involvement in the different phases of the demonstrator, PISA-like problem solving questions have been introduced, in order to measure the students' competence and gained abilities from the educational intervention.

\section{Description of the "HY.P.A.T.I.A" data analysis tool}

The HYPATIA demonstrator uses the embedded HYPATIA [10], [11] on-line tool. HYPATIA is an event visualization tool that uses real data from the ATLAS experiment, namely offers a graphical representation of the products of proton collisions. The students using the event display can interactively analyse the data collected almost in real-time. This allows them to take advantage of the LHC data from CERN without having to leave their classroom! The students learn how to identify particles and their interactions, and learn how the gigantic state-of-the-art ATLAS detector works. They also get a glimpse at how the data analysis that led to the discovery of the Higgs boson is performed.

The on-line version of HYPATIA has been developed since 2010 by the University of Athens. It complements the off-line version [12] which is used by the International Masterclasses [13] since 2008 by thousands of high-school students in all continents each year. The on-line version is simpler to use, yet contains all the functionalities necessary for performing the various scenaria associated with the different exercises. It includes four different parts each with an increasing level of complexity and difficulty.

The best practice for introducing HEP to 16-18 year old students is a combination of lectures, virtual tours to specific experiments and hands-on experience with HYPATIA. This combination has taken the form of the so called "mini masterclasses" which are half-day workshops, taking place locally at the interested schools. During the last five years the "mini masterclasses" were run in about 100 schools across the Greece (Fig. 2) as well as in other European sites (CERN, Ireland etc.). 


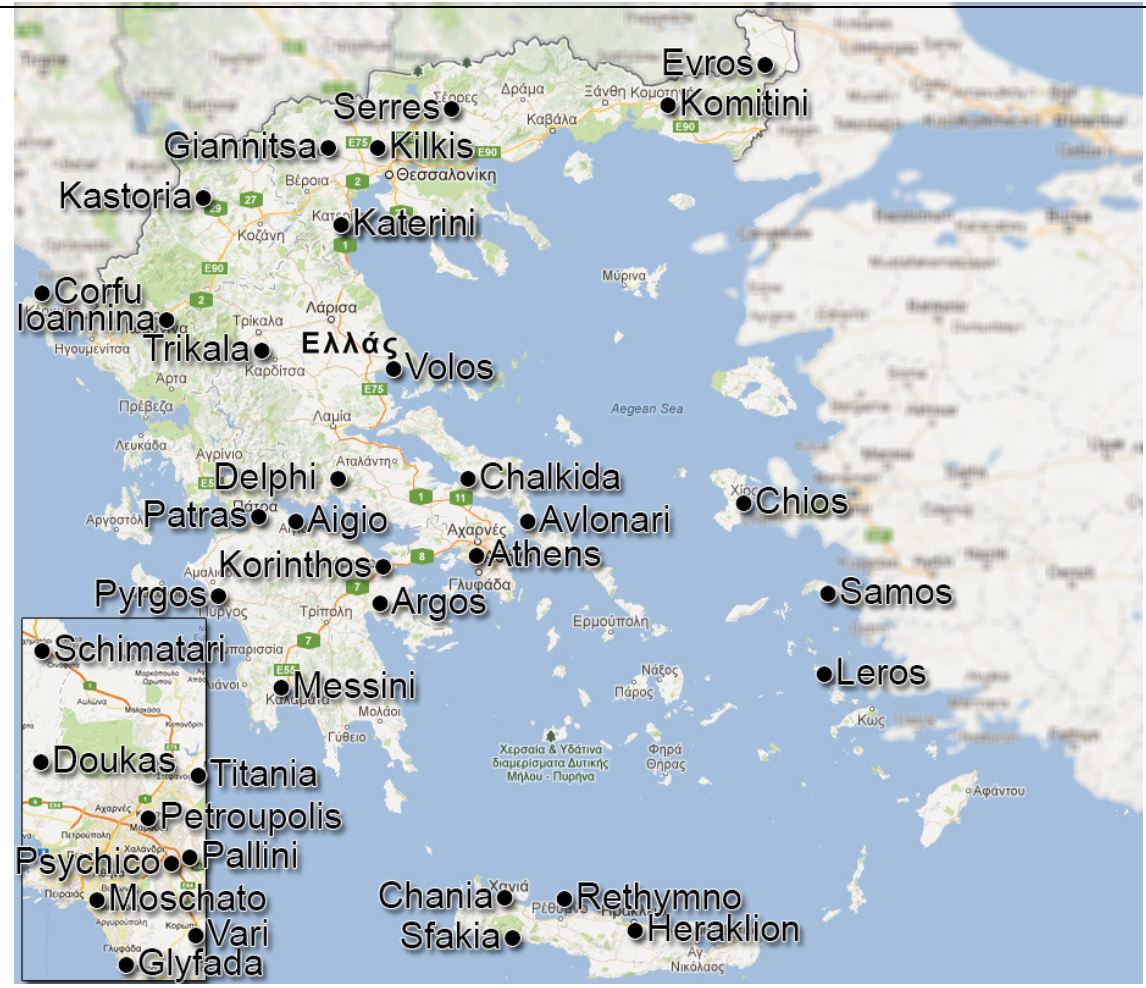

Figure 2. Map of Greece showing the schools where the "mini masterclasses" took place during the last few years.

Fig 3 shows students at the Giannitsa city (northern part of Greece) while analysing the ATLAS events during a "mini masterclass" last summer which was part of a week-long training organized by the Greek Physical Society for high performing local students. 


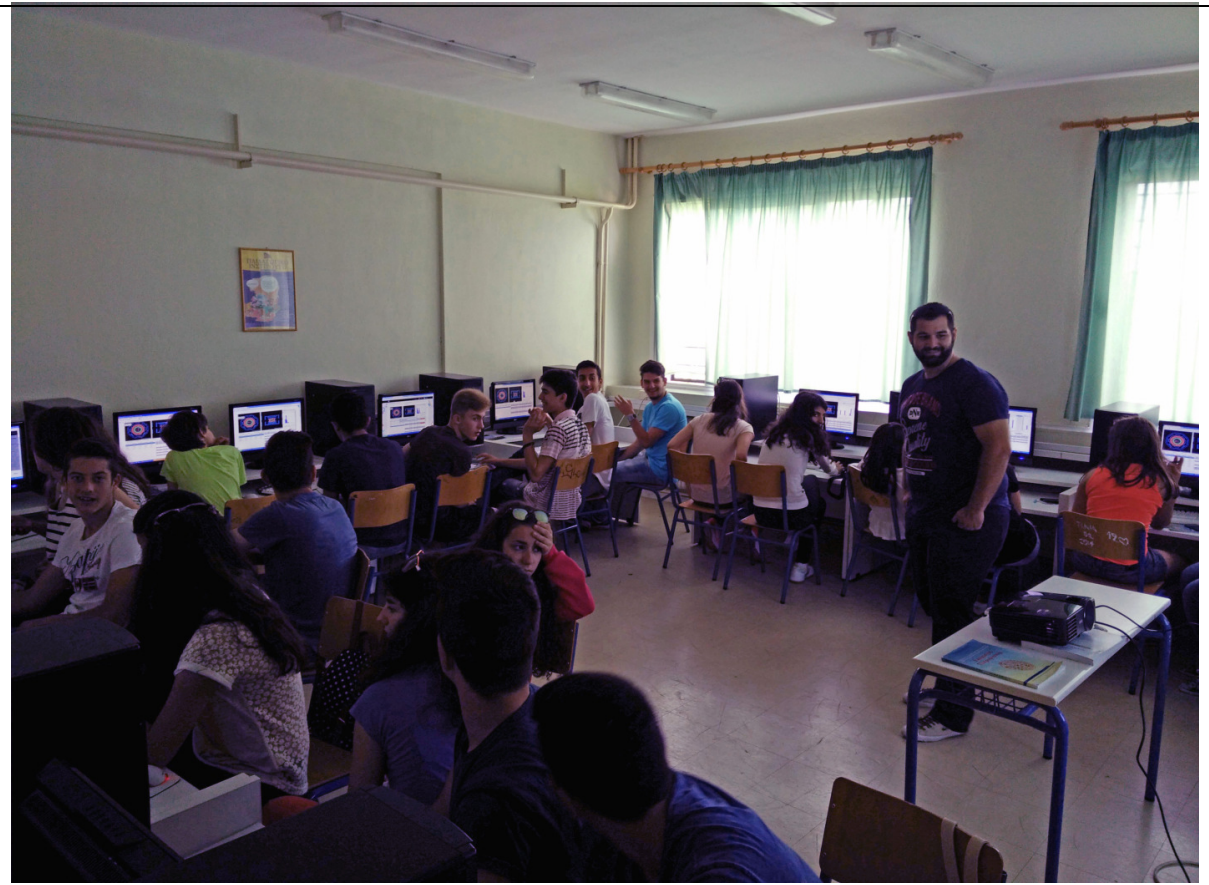

Figure 3. Students at Giannitsa city working on the HYPATIA demonstrator.

During last semester the students' assessment has been tested in eight locations in Greece with about 250 students from 35 different schools. The preliminary results show that the students out-perform the corresponding Pisa assessment questions' scores in OECD countries.

\section{The HYPATIA tool for a university laboratory}

Besides the efforts to raise enthusiasm and motivate high school students, it was realized that the university laboratory exercises should include the students' involvement in most modern top research, besides the so called "classical" experiments. The students during their lab courses should perform activities which are directly connected with the most advanced research at CERN. The corresponding involvement should be at a higher level than the hands-on exercises for younger ones.

Since 2010 the HYPATIA tool is used in a lab course of the University of Athens for fourth year undergraduate physics students, majoring in HEP [14]. Each year about 50 students take part in the three hour long course performing all steps of the HYPATIA demonstrator (introductory material, become familiar with the tool, identify particles, reconstruct $\mathrm{Z}$ bosons in leptonic decay modes and search the Higgs boson). In addition, at the end of the lab, they have to hand-in a homework paper where they try to answer advanced questions which are related to the decays modes, widths, masses, experimental resolution and basic statistical analysis tools.

Recently an additional three hour long course has been developed which follows the above -mostly visual- inspection of events. The designed additional lab aims to permit the students to perform an in depth analysis of large samples of events. The students after visual inspection of a few tens of events, run HYPATIA in the so called "batch mode" (Fig. 4). 


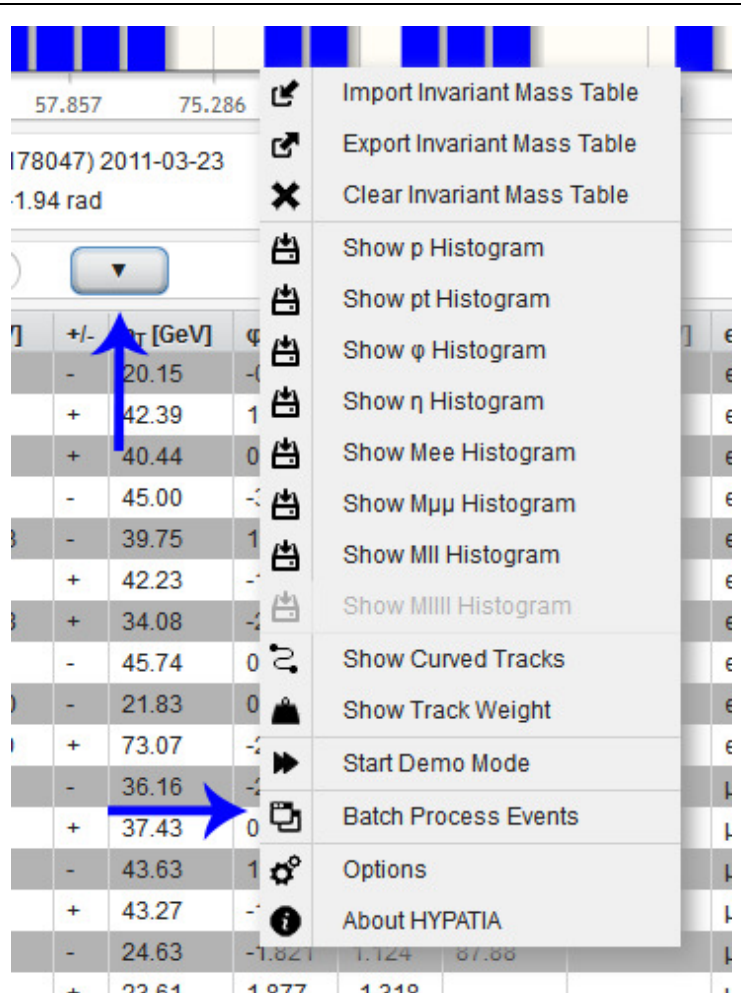

Figure 4. Drop-down HYPATIA menu for "Batch mode" running

They are able to analyse simultaneously two large samples of events and optimize the cuts for the $\mathrm{H} \rightarrow 41$ decay searches in a way which is very similar to the one the researchers employ and which led to the Higgs boson discovery in the summer of 2012. They can analyse either two-lepton samples to look for resonances such as the $J / \psi, \Upsilon, Z$ etc. or four-lepton samples to look for the Higgs boson. In the following the Higgs analysis is described.

The samples used can be either Monte-Carlo ones (signal and different backgrounds) or data ${ }^{a}$. The backgrounds include the $\mathrm{ZZ}^{(*)}$ irreducible one, as well as $\mathrm{Z}+$ jets $\left(\mathrm{Z}^{\mathrm{b} b}\right)$ reducible one. The students have to realize that certain kinematical distributions of the signal are different from the corresponding ones of the background and that this is the main methodology for applying cuts in order to reduce the background and make a discovery.

They start the investigation with the Monte Carlo samples in order to optimize the cuts. The samples -in order to reduce their size- have undergone some filtering cuts: the values of the $\mathrm{p}_{\mathrm{T}}$ of each of the four leptons should be larger than $6 \mathrm{GeV}$ and the invariant mass of their two or four lepton combinations larger than $2 \mathrm{GeV}$. The variables available by the exercise where the cuts could be applied are the following:

- The momenta of all pre-selected four leptons $\mathrm{p}_{\mathrm{T} 1,2,3,4}$

- $\mathrm{m}_{12}$ : the mass of the lepton pair (opposite sign, same flavour) closest in value to the $\mathrm{Z}$ mass

- $\mathrm{m}_{34}$ : the mass of the next lepton pair (opposite sign, same flavour) closest in value to the $\mathrm{Z}$ mass

- The largest impact ${ }^{\mathrm{b}}$ parameter out of the leptons in the event (divided by its error). Separately for muons $\left(\mathrm{d}_{0 \mu}\right)$ and electrons $\left(\mathrm{d}_{0 \mathrm{e}}\right)$.

${ }^{a}$ Very recently the ATLAS management gave permission to use for outreach purposes $1 \mathrm{fb}^{-1}$ of the 2012 data which will eventually appear in the opendata portal for all LHC experiments [15] 
- Distance of the lepton vertex $\left(\mathrm{z}_{\mathrm{vtx}}\right)$ from the beam crossing vertex $\left(\mathrm{z}_{0}\right)$ along the $\mathrm{z}$ (beam) axis

- The largest isolation ${ }^{c}$ parameter out of the leptons in the event.

- Cut on the minimum or maximum invariant mass of the four lepton combination.

The table of cuts is shown on Fig. 5 together with some pre-set values for each cut (which should be optimized during the analysis process).

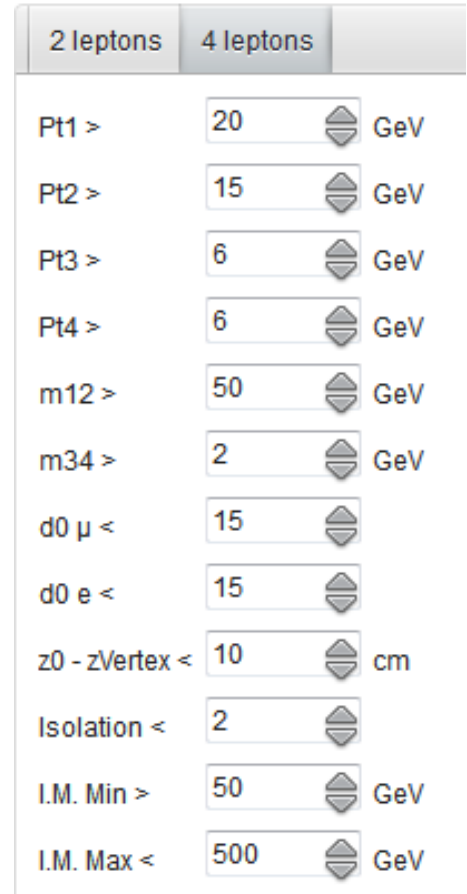

Figure 5. Tables of the variables available for optimization (together with some indicative values).

${ }^{\mathrm{b}}$ Impact parameter is the vertical distance of the secondary lepton vertex from the interaction point

(primary vertex)

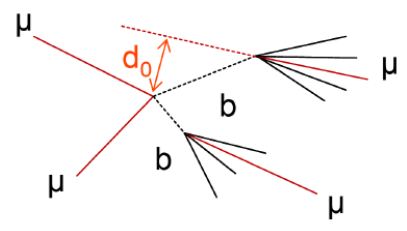

${ }^{c}$ The Isolation parameter is calculated by taking a cone of about $30^{\circ}$ opening angle, adding all the $\mathrm{p}_{\mathrm{T}} \mathrm{S}$ of tracks inside the cone (subtracting the lepton under consideration) and dividing by the $\mathrm{p}_{\mathrm{T}}$ of the

track.

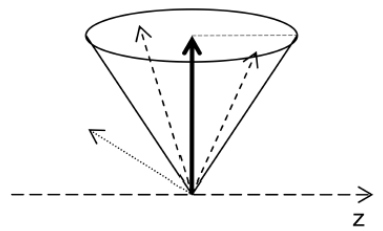


The students use the so called "N-1" technique to optimize each cut. Namely they examine the distribution (histogram) of the variable under investigation having applied all the other cuts, except the cut under optimization. Its value is then decided examining the corresponding distributions for signal and background as well as the distribution of the statistical significance ${ }^{d}$ of the signal. Such an example is given on Fig. 6. The optimal value of the cut is the point where the significance is highest. After fixing the value of the particular cut, the students proceed to the next one until they optimize all (or a subset) of them. In subsequent iterations, the students start with the new values of the cuts and re-optimize them one by one in order to arrive at a new optimal set.

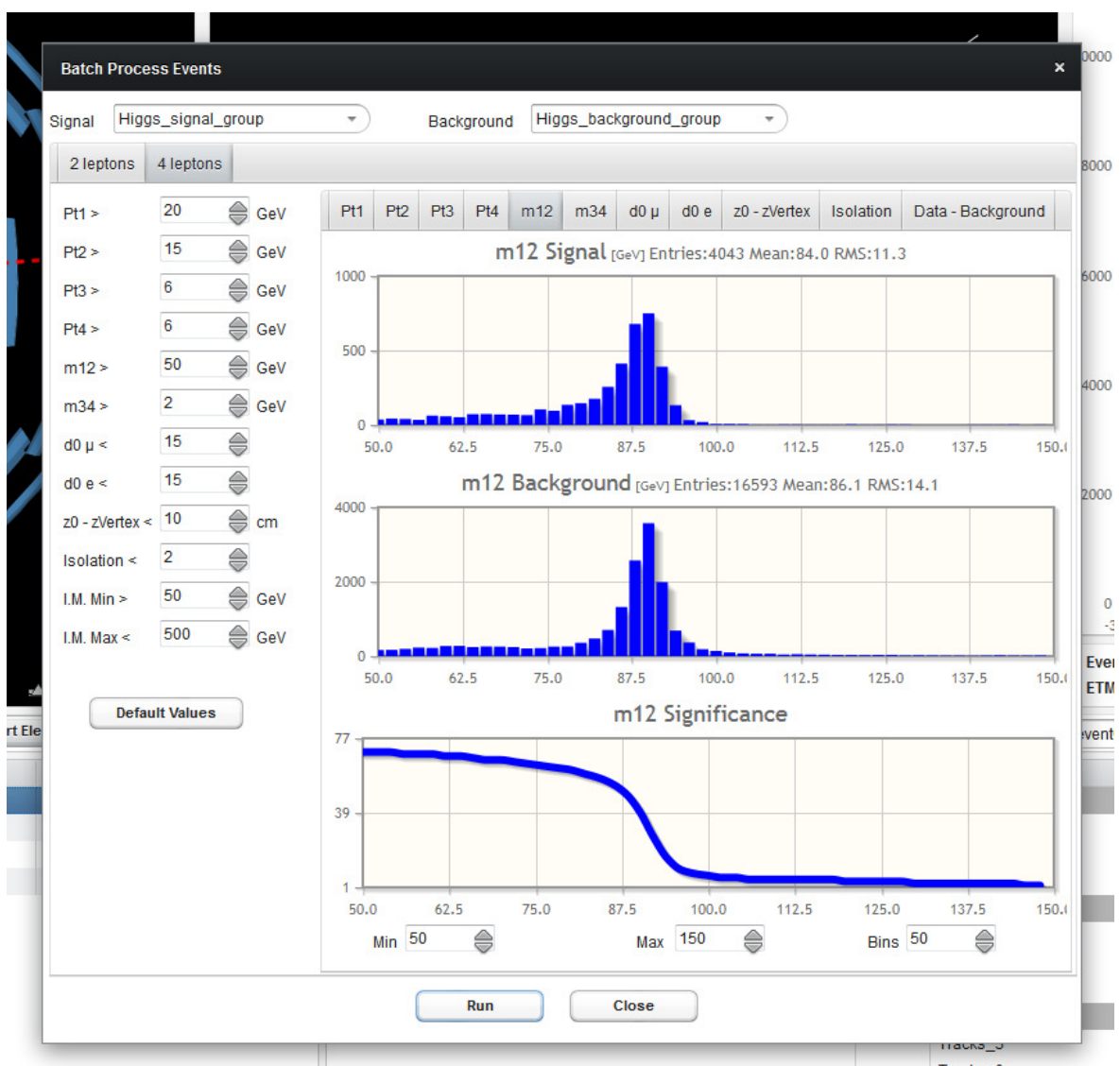

Figure 6. Histogram of a specific kinematic parameter $\left(\mathrm{m}_{12}\right)$. The top distribution is the distribution from the Monte-Carlo signal sample, the middle is the corresponding Monte Carlo background distribution (ZZ ${ }^{(*)}$ plus $Z+$ jets) and the bottom one is the statistical significance derived from the above two distributions as a function of the $\mathrm{m}_{12}$ value.

$\mathrm{d}$ The signal significance is defined as:

significance $=\sqrt{2 *\left((S+B) * \ln \left(1+\frac{S}{B}\right)-S\right)}$ 
The point where the students are instructed to stop the iterations is determined by looking at a narrow region around the Higgs boson mass in the four lepton invariant mass where they should maximize, after a number of iterations, the value of the signal significance.

The present "batch mode" processing exercise can also be applied to Z' $\rightarrow 21$ Monte-Carlo sets for the Z' cut optimization. In this case the investigation is simpler and quicker because the students optimize the cuts for two leptons only, thus having fewer variables to iterte on.

The $\mathrm{H} \rightarrow 41$ exercise is been implemented during the fall 2015 semester in the University of Athens and Birmingham advanced students' lab.

\section{The Chania science fair event}

In an effort to get the general public aware about CERN and HEP in general, a full day science fair was organized in the context of the ICNFP 2015 conference. The event took place on the 28th August in Chania, which is the second largest city in Crete, about 40km away from Kolymbari. It was organized for the second consecutive year and took place at the premises of the Chania Sailing club, a $16^{\text {th }}$ century Venetian dockyard beautifully restored. Several conference participants took part and guided the visitors to the world of top level research. Simple experiments or Masterclasses (ATLAS and ALICE) were set up so the visitors could get hands-on experience. The simple experiments (donated by Quarknet) were "the conservation of momentum, rolling with Rutherford, probe matter, quark workbench and W signatures" [16]. They were all set up on long benches (see Fig. 7) where the public could circulate around and get involved one way or the other. At the same time, several colleagues were present to answer questions made by the public. The event lasted until late in the afternoon and there were about 150 visitors of various nationalities but mostly parents with their children.

In the evening at the same place a public lecture by Prof. E. Kiritsis of the University of Crete with title "Microscopic black holes: a new window on the universe" took place and was very well attended. In summary, the science fair had a great success and will be repeated next year for ICNFP 2016. 


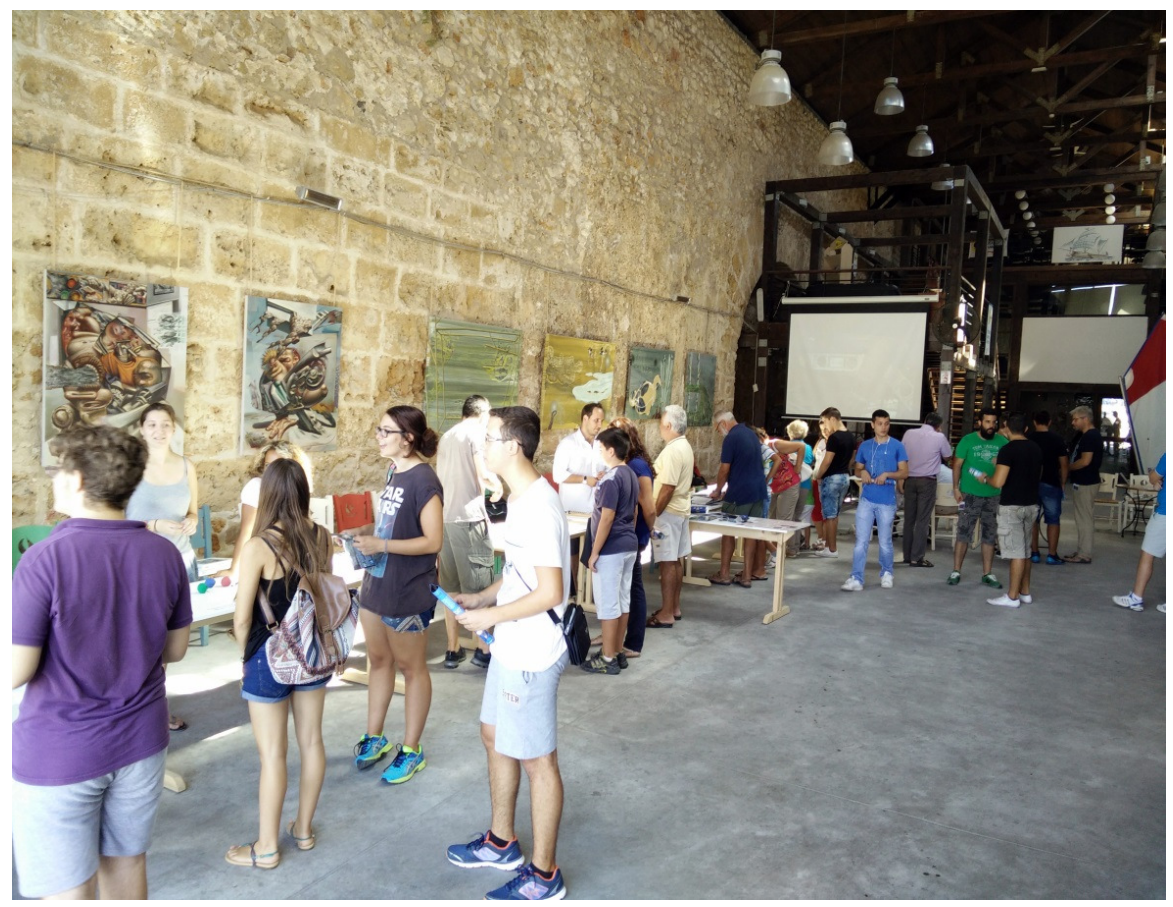

Figure 7. The Chania science fair set up at the Sailing Club.

\section{Conclusions}

Recent hands-on activities, development and testing of innovative applications towards the goal of introducing HEP to the schools and universities are reviewed. A large number of resources developed by European Outreach programs exist on the web and these have recently been enriched by applications for university students. This way many thousands of students as well as the general public can get acquainted to the top level research of CERN, and grow a real enthusiasm for it.

\section{ACKNOWLEDGEMENT}

This work was partially funded by the European Union in the context of the ISE project (Grant Agreement no. 325123) under the CIP Framework program. This document does not represent the opinion of the European Union, and the European Union is not responsible for any use that might be made of its content.

\section{References}

[1] http://www.learningwithATLAS-portal.eu/

[2] http://pathway-project.eu/

[3] http://www.discoverthecosmos.eu/

[4] http://www.go-lab-project.eu/

[5] http://inspiring-science-education.eu/

[6] http://www.portal.discoverthecosmos.eu/

[7] http://www.oecd.org/pisa/pisaproducts/46962005.pdf 
[8] http://opendiscoveryspace.eu/

[9] http://portal.opendiscoveryspace.eu/edu-object/hypatia-demonstrator-english-v2-828289

[10] C. Kourkoumelis, \& S. Vourakis, (2014) Hypatia-an online tool for ATLAS event visualization.

Physics Education, 49, 21-32

[11] http://hypatia.iasa.gr/

[12] http:/hypatia.phys.uoa.gr/

[13] http://physicsmasterclasses.org/

[14] R.M. Barnett, K.E. Johansson, C. Kourkoumelis, L. Long, J. Pequenao, C. Reimers, \& P. Watkins, (2012) Learning with ATLAS experiment at CERN, Physics Education, 47, 28

[15] http://opendata.cern.ch/

[16] https://quarknet.i2u2.org/data-portfolio 